\title{
Evaluation of Efficacy of Hypertonic Saline and Mannitol in Combination in Patients with a Traumatic Brain Injury
}

\author{
Taylor Schwebke, Pharm.D ${ }^{1 *}$, Marc McDowell, Pharm.D, Lina Piech, Pharm.D, Robert Mokszycki, Pharm.D and Ellen Omi, MD \\ Advocate Christ Medical Center, USA
}

${ }^{\star}$ Correspondence Author: Taylor Schwebke, 1438 W Addison St, Apt 1, Chicago, IL 60613; E-mail: taylor.schwebke@gmail.com

Received: September 02, 2019; Accepted: September 15, 2019; Published: October 03, 2019;

\begin{abstract}
Title: Evaluation of Efficacy of Hypertonic Saline and Mannitol in Combination in Patients with a Traumatic Brain Injury

Background: Traumatic Brain Injury (TBI) is a major cause of disability and death. In 2013, an estimated 2.5 million emergency department visits were related to TBI. In current practice, hyperosmolar therapy such as bolus doses of intravenous mannitol $20 \%$ and sodium chloride $3 \%$ are commonly used as single agents for the treatment of Cerebral Edema And Intracranial Pressure (ICP) reduction. The Brain Trauma Foundation guidelines do not recommend a preferred agent, nor do they comment on the use of these agents in combination. Currently, there are no published studies evaluating combination hyperosmolar therapy for ICP reduction. The primary objective of this study is to evaluate the efficacy of bolus doses of intravenous mannitol $20 \%$ and sodium chloride $3 \%$ utilized as monotherapy or in combination in patients with TBI.
\end{abstract}

Methods: This single-center retrospective study identified cases using a medication usage report generated through the electronic medical record at Advocate Christ Medical Center. Subjects 14 years and older admitted to the trauma service following a diagnosis of TBI who received an intravenous bolus of mannitol 20\% and/or sodium chloride 3\% from August 1, 2013 through August 1, 2018 were included. Subjects without known trauma confirmed by radiographic imaging, those who received a sodium chloride $3 \%$ continuous infusion, pregnant patients, and those who expired within twentyfour hours of admission were excluded. The following data points were collected: age, weight, gender, race, serum creatinine, serum sodium, serum osmolality, mean arterial pressure, initial Glasgow Coma Score (GCS), diagnostic imaging, presence of cerebral edema and size of midline shift on computed tomography, dose and frequency of hyperosmolar agents, neurosurgical intervention, vasopressor requirements, intravenous fluids, Hospital And Intensive Care Unit (ICU) Length Of Stay (LOS). All data was recorded without patient identifiers, maintained confidentially, and was analyzed using descriptive and inferential statistics.

Results: A total of 1000 patients were screened of which 176 met the inclusion/exclusion criteria. In-hospital mortality was experienced by 6 of 24 patients in the combination group compared to 18 of 152 patients in the monotherapy group $(\mathrm{p}=0.08)$. Statically significant reductions were seen in need for neurosurgical intervention $(p=0.04)$, vasopressor utilization $(p=0.03)$, and ICU LOS $(p=0.02)$ demonstrating a benefit of monotherapy over combination. No difference was seen in laboratory values or vitals measurements.

Conclusion: Combination therapy was associated with a trend towards increase mortality compared to monotherapy use.

Keywords: hypertonic saline, intracranial pressure, mannitol, Traumatic brain injury

\section{Introduction}

Traumatic Brain Injury (TBI) is a major cause of disability and death in the United States. According to the Center for Disease Control and Prevention there are approximately 2.5 million emergency department visits related to TBI each year. These injuries resulted in 282,000 hospitalizations and 56,000 deaths.[1] in 2010, the total economic impact of TBI was estimated to be $\$ 76.5$ billion.[2]

A TBI is defined as a bump, blow, or jolt to the head that disrupts the normal function of the brain commonly caused by falls, blunt trauma, and motor vehicle crashes. The severity of injury ranges from mild to severe.[1] The Glasgow Coma Scale is a validated tool used to assess the initial severity of brain injury. A GCS score of $13-15$ is considered mild injury, $9-12$ is considered moderate injury, and 8 or less is considered severe TBI.[3] For those who survive, the effects of a
TBI can be temporary or leave an individual with permanent deficits. Effects of TBI can include impaired thinking, memory, movement, emotional, and social functioning.[1] These issues not only affect individuals but can have lasting impacts on families, communities, and the healthcare system.

A primary mechanism of poor neurologic outcomes in TBI stems from cerebral edema and subsequent increase in Intracranial Pressure (ICP). Tissue swelling from the injury can increase pressure inside of the skull, alter blood perfusion, and cause additional damage to the brain. A normal ICP ranges from $5-15 \mathrm{mmHg}$.[4] A sustained ICP of greater than $20 \mathrm{mmHg}$ for greater than five minutes is associated with increased morbidity and mortality.[5]

In current practice, osmotic therapy with intravenous mannitol or hypertonic saline is used in symptomatic patients with cerebral 
edema or ICP elevation. Hyperosmolar therapy creates an osmolar gradient across the blood-brain barrier and also causes a reduction in blood viscosity by decreasing red blood cell rigidity and cohesiveness improving microvascular circulation. Mannitol, a sugar alcohol, works as a potent osmotic diuretic. It is administered in Intravenous Piggyback (IVPB) bolus doses of $0.25-1 \mathrm{mg} / \mathrm{kg}$ every 6-8 hours. Adverse effects include hypovolemia, hypotension, acute kidney injury and metabolic disturbances. Hypertonic saline is supplied in a variety of concentrations, the $3 \%$ concentration is dosed $250-500 \mathrm{~mL}$ (128-256 mEq sodium) IVPB bolus every 4-6 hours. Adverse effects include hypervolemia, hypertension, and electrolyte disturbances. [6] The two therapies are not mutually exclusive and may be used in combination. Guidelines from the Brain Trauma Foundation make no recommendation on a preferred agent, nor do they comment on the use of combination therapy.[7] Currently, there are no published studies evaluating combination hyperosmolar therapy for ICP reduction. The purpose of this study is to evaluate the safety and efficacy of the combination of mannitol and hypertonic saline versus each agent used as monotherapy.

\section{Methods}

\section{Patient population and setting}

The single study site was a large, urban level 1 trauma center located on the Southside of Chicago, Illinois. Eligible patients were identified using a medication usage report generated through the electronic medical record for those admitted from August 1, 2013 through August 1, 2018. Screening for further inclusion and data collection was completed using the financial identification number of each patient. This study was approved by the local Institutional Review Board.

\section{Inclusion/exclusion}

Patients were included if they were at least 14 years old. Patients also must have been admitted to the trauma service, have radiographic imaging confirming TBI, and receive at least one of the study drugs to be included. Patients were excluded if they were less than 14 years old, pregnant, died within 24 hours of admission, did not have a history of recent trauma or radiographic evidence of a TBI, or did not receive study drug as a bolus.

\section{Outcomes}

The primary outcome of this study was incidence of inhospital mortality. Secondary outcomes included the occurrence of neurosurgical evacuation, change in serum sodium, creatinine, and osmolality, Mean Arterial Pressure (MAP), and hospital and ICU LOS. Change in baseline laboratory measurements was defined as change from prior to 24 hours after the first dose of hyperosmolar therapy. Change in baseline MAP measurements was defined as change from prior to 30 minutes after the first dose of hyperosmolar therapy.

\section{Statistical Analysis}

A sample size of 800 patients was calculated to achieve $80 \%$ power to detect a difference of 10 percentage points between groups for the primary outcome of in-hospital mortality. Demographic and baseline data points were calculated as mean or median dependent upon their normality as assessed by the Shapiro-Wilk Statistic. Statistical analysis of all endpoints was done by combining the HTS and MAN group to make a monotherapy group that was tested against combination. ChiSquare tests were performed for categorical data and Mann-Whitney $\mathrm{U}$ tests were performed for continuous data. All tests were two-tailed and a p-value of 0.05 was considered statistically significant in all analyses.

\section{Results}

A total of 1000 patients were screened for inclusion in this study and 176 patients met the criteria. A total of 81 patients received only sodium chloride $3 \%$ IVPB bolus, 71 patients received only mannitol 20\% IVPB bolus, and 24 patients received a combination of these two agents in scheduled alternating IVPB bolus form. Patient demographics of the study groups did not differ statistically (Table 1). The typical patient was middle aged, male, of Caucasian or African American decent. Patients who received combination therapy tended to be younger, were more likely male, and more likely African American. The median dose of HTS for all patients who received a dose was $250 \mathrm{~mL}$ (128mEq). The average mannitol dose was $0.8 \mathrm{~g} / \mathrm{kg}$ for all patients who received a dose. Most patients received 1-2 days of therapy as denoted by number of doses received.

Table 1. Patient Demographics

\begin{tabular}{|l|c|c|c|}
\hline & HTS (n=81) & MAN (n=71) & HTS+MAN (n=24) \\
\hline Age (years), mean & 53.7 & 50.8 & 41.2 \\
\hline Male, $n(\%)$ & $63(76.5)$ & $54(76.1)$ & $20(83.3)$ \\
\hline Weight (kg), mean & 84.1 & 92.8 & 81.4 \\
\hline Race, $n(\%)$ & & & \\
\hline Caucasian & $37(45.7)$ & $36(50.7)$ & $8(33.3)$ \\
\hline African American & $29(35.8)$ & $33(46.5)$ & $14(58.3)$ \\
\hline Hispanic & $10(12.3)$ & 0 & $1(4.2)$ \\
\hline Unknown & $5(6.2)$ & $2(2.8)$ & $1(4.2)$ \\
\hline
\end{tabular}

Characteristics and severity of TBI at initial presentation were collected and are displayed in table 2. Patients who received combination therapy tended to have lower GCS scores than those receiving monotherapy, however this difference was not statistically significant. All patient groups were similar in terms of rates of cerebral edema and midline shift as well as imaging findings of types of hemorrhage.

Results of the primary and secondary outcomes of the study are shown in table 3. For the primary endpoint of mortality, a nonsignificant relationship was identified between combination therapy and mortality, with $11.8 \%$ of those on hypertonic saline or mannitol expiring after 24 hours as compared to $25.0 \%$ of those on hypertonic saline and mannitol expiring after 24 hours $(\mathrm{p}=0.08)$.

For the secondary endpoint of ICU LOS, a significant relationship was identified with those who received either hypertonic saline or 
Taylor Schwebke (2019) Evaluation of Efficacy of Hypertonic Saline and Mannitol in Combination in Patients with a Traumatic Brain Injury

mannitol having a median ICU LOS of 6.0 as compared to those who received both hypertonic saline and mannitol having a median ICU LOS of $9.1(\mathrm{p}=0.02)$. A total $7.2 \%$ of those on hypertonic saline or mannitol received vasopressor therapy, compared to $20.8 \%$ of those on hypertonic saline and mannitol ( $\mathrm{p}=0.03)$. Neurosurgical intervention was needed on $30.3 \%$ of those on hypertonic saline or mannitol as compared to $45.8 \%$ of those on combination therapy $(p=0.04)$. No difference was seen in fluid bolus requirements $(\mathrm{p}=0.43)$ or hospital LOS $(p=0.55)$. None of the baseline values or repeat laboratory values were found to be significantly different between the groups. Furthermore, no significant result was found on MAP measurements $(\mathrm{p}=0.10)$.

A post-hoc sub analysis was performed on the primary endpoint and the hypertonic saline group (Table 4). Hypertonic saline had statistically lower mortality compared to combination therapy $(\mathrm{p}=0.03)$. However, it was not statically superior to mannitol when compared directly $(\mathrm{p}=0.19)$.
Table 2. Injury Severity and Characteristics. Values are median [interquartile range] except those specified with $\dagger$ are mean \pm standard deviation

\begin{tabular}{|l|c|c|c|}
\hline & $\begin{array}{c}\text { HTS } \\
(\mathbf{n = 8 1})\end{array}$ & MAN (n=71) & $\begin{array}{c}\text { HTS+MAN } \\
(\mathbf{n = 2 4})\end{array}$ \\
\hline GCS & $12[7-15]$ & $12[7-15]$ & $9.2 \pm 4 \dagger$ \\
\hline $\begin{array}{l}\text { Presence of Cerebral Edema, } \\
n(\%)\end{array}$ & $35(43.2)$ & $36(50.7)$ & $11(45.8)$ \\
\hline Presence of Midline Shift, $n(\%)$ & $26(32.1)$ & $42(59.2)$ & $11(45.8)$ \\
\hline Imaging, $n$ (\%) & $53(65.4)$ & $48(67.6)$ & $16(66.7)$ \\
\hline Subdural Hemorrhage & $47(58.0)$ & $34(47.9)$ & $15(62.5)$ \\
\hline Subarachnoid Hemorrhage & $30(37.0)$ & $24(33.8)$ & $7(29.2)$ \\
\hline Intraparenchymal Hemorrhage & $3(3.7)$ & $6(8.5)$ & $2(8.3)$ \\
\hline Epidural Hemorrhage & $4(4.9)$ & $2(2.8)$ & 0 \\
\hline Diffuse Axonal Hemorrhage & $2(2.5)$ & $1(1.4)$ & 0 \\
\hline Intracerebral Hemorrhage & \multicolumn{3}{|c|}{} \\
\hline
\end{tabular}

Table 3. Primary and secondary study outcomes. Values are median [interquartile range] except those specified with $\dagger$ are mean \pm standard deviation. $*$ indicates significance at $\mathrm{p}=0.05$.

\begin{tabular}{|c|c|c|c|c|}
\hline & \multicolumn{2}{|c|}{ Monotherapy } & \multirow{2}{*}{$\begin{array}{c}\text { Combination } \\
\text { HTS+MAN }(n=24)\end{array}$} & \multirow[t]{2}{*}{ P-value } \\
\hline & HTS $(n=81)$ & MAN $(n=71)$ & & \\
\hline Mortality, $n(\%)$ & $7(8.6)$ & $11(15.5)$ & $6(25)$ & 0.08 \\
\hline Neurosurgical Procedure sPPrprIntervention, $n(\%)$ & $14(17.3)$ & $32(45.1)$ & $11(45.8)$ & $0.04 *$ \\
\hline Craniotomy, $n(\%)$ & $10(12.4)$ & $18(25.3)$ & $3(12.5)$ & \\
\hline Craniectomy, $n(\%)$ & $4(4.9)$ & $13(18.3)$ & $8(33.3)$ & \\
\hline Both, $n(\%)$ & 0 & $1(1.4)$ & 0 & \\
\hline Vasopressors Used, $n(\%)$ & $6(7.4)$ & $5(7)$ & $5(20.8)$ & $0.03 *$ \\
\hline Fluid Bolus Used, $n(\%)$ & $16(19.8)$ & $23(32.4)$ & $8(33.3)$ & 0.43 \\
\hline Hospital LOS (days) & $10.9[6.2-16.4]$ & $12.0[7.6-18.6]$ & $13.8 \pm 7.4 \dagger$ & 0.55 \\
\hline ICU LOS (days) & $6.0[3-9]$ & $5.0[3-10]$ & $9.1[5.5-11.6]$ & $0.02 *$ \\
\hline Initial Serum Sodium $(\mathrm{mmol} / \mathrm{L})$ & $139( \pm 3.9) \dagger$ & $139[137-141]$ & $138.5( \pm 3.7) \dagger$ & 0.63 \\
\hline Repeat Serum Sodium (mmol/L) & $142.7( \pm 6.3) \dagger$ & $140[138-144]$ & $142.5[140-146.5]$ & 0.13 \\
\hline Initial Serum Creatinine $(\mathrm{mg} / \mathrm{dL})$ & $0.9[0.8-1.2]$ & $1.0[0.8-1.2]$ & $0.9[0.8-1.1]$ & 0.61 \\
\hline Repeat Serum Creatinine (mg/dL) & $1.0[0.6-1.0]$ & $0.8[0.7-1.1]$ & $0.8[0.7-1.2]$ & 0.63 \\
\hline Low Serum Osmolality (mOsm/kg) & $299.9( \pm 12.9) \dagger$ & $293.8( \pm 13.4) \dagger$ & $303.6( \pm 21.6) \dagger$ & 0.11 \\
\hline High Serum Osmolality (mOsm/kg) & $302[293-322]$ & $314.5[304-328]$ & $317.2( \pm 20.5) \dagger$ & 0.56 \\
\hline Initial MAP (mmHg), mean & 92.7 & 97.0 & 97.4 & \\
\hline Repeat MAP, $n(\%)$ & & & & 0.10 \\
\hline No Change & $57(70.4)$ & $46(64.8)$ & $12(50)$ & \\
\hline Increase $>10 \mathrm{mmHg}$ & $11(13.6)$ & $11(15.5)$ & $3(12.5)$ & \\
\hline Decrease $<10 \mathrm{mmHg}$ & $13(16)$ & $14(19.7)$ & $9(37.5)$ & \\
\hline
\end{tabular}


Table 4. Primary Outcome Sub Analysis. * indicates significance at $\mathrm{p}=0.05$.

\begin{tabular}{|l|l|l|l|}
\hline \multicolumn{4}{|c|}{ Sub analysis: Sodium Chloride 3\% vs Combination } \\
\hline & HTS (n=81) & $\begin{array}{c}\text { HTS+MAN } \\
(\mathbf{n}-24)\end{array}$ & P-value \\
\hline Mortality, $n(\%)$ & $7(8.6)$ & $6(25)$ & $0.03 *$ \\
\hline
\end{tabular}

\begin{tabular}{|l|l|l|l|}
\hline \multicolumn{4}{|l|}{ Sub analysis: Sodium Chloride 3\% vs Mannitol } \\
\hline & HTS (n=81) & MAN (n-71) & P-value \\
\hline Mortality, $n(\%)$ & $7(8.6)$ & $11(15.5)$ & 0.19 \\
\hline
\end{tabular}

\section{Discussion}

In this retrospective study, TBI patients were administered hyperosmolar therapy to decrease intracranial pressure. We found a trend towards a decrease in in-hospital mortality associated with monotherapy use with either sodium chloride $3 \%$ or mannitol $20 \%$ compared to combination therapy. The dosing of both agents was in accordance with current practice and first doses were often administered immediately after the patient presented to the emergency department with traumatic injuries. All groups were similar in demographic and injury characteristics with the typical patient presenting with a moderate TBI.

We predicted that the mannitol group may experience more adverse events such as acute kidney injury and hypotension. However, we did not find these results as serum sodium, creatinine, osmolality and MAP measurements did not significantly change 24 hours after the first dose of hyperosmolar therapy. Previous literature may overestimate the detrimental effects of hyperosmolar therapy including mannitol, or the initial dosing used in our cohort may be low enough to avoid these effects. The patients included in our study were primarily young, otherwise healthy adults who may better tolerate the medications compared to older adults with a higher likelihood of comorbid renal and cardiovascular conditions.

We found that monotherapy was associated with a decrease in neurosurgical interventions. This decrease likely led to a significant decrease in ICU and overall hospital LOS. These reductions result in a great cost savings and imply better outcomes for patients. The sub analysis of our primary outcome revealed a statically significant reduction in mortality with the use of sodium chloride $3 \%$ monotherapy compared to combination therapy with mannitol. However, sodium chloride $3 \%$ was not significantly better than monotherapy with mannitol. Sodium chloride $3 \%$ may be the preferred agent for ICP reduction after TBI, but further research is needed in this area. Based on the results of the primary outcome being nonsignificant, it was determined that mannitol alone would not be statistically superior than combination therapy at lowering in-hospital mortality as the composite of mannitol and hypertonic saline was not significant.

Our study suffered from several limitations. This data was collected from a single center in a retrospective manner. It did not meet power for the primary endpoint. The combination therapy group had a small sample size of 24 patients and these patients had a lower initial GCS score. This could mean this was a more critically ill group and thus more likely to need advanced therapies, have poorer outcomes and expire. However, none of the baseline data points were statistically significant between groups. We did not collect data on other injuries beyond those of the head which often contribute to patient death from traumatic causes. There was also variance in the dosing of hyperosmolar therapy, and timing of blood pressure and laboratory measurements. Lastly, our institution does not routinely use ICP monitors, consequently our efficacy data was purely symptom based.

\section{Conclusion}

Combination therapy was associated with a trend towards increased mortality compared to monotherapy use. Compared to combination therapy, subjects treated with monotherapy had a statistically significant reduction in ICU LOS, need for neurosurgical evacuation of hemorrhage, and vasopressor therapy to maintain MAP goals.

As this is the first published study evaluating the use of combination hyperosmolar therapy in TBI, further evaluation is needed. Specifically, the evaluation of a continuous infusion of hypertonic saline in addition to mannitol IVPB maybe an area of promise. Additionally, a prospective study would be able to set standard dosing and monitoring regimens. A randomized control study of monotherapy versus combination therapy for hyperosmolar agents in TBI would allow the establishment of a causal association of these therapies with outcomes such as mortality, ICU LOS, and need for neurosurgical interventions.

\section{References}

1. Taylor CA, Jeneita M Bell, Matthew J Breiding, Likang Xu (2017) Traumatic Brain Injury - Related Emergency Department Visits, Hospitalizations, and Deaths - United States, 2007 and 2013. MMWR. US Department of Health and Human Services 66: 1-16.

2. Faul M, Likang Xu, Marlena M. Wald, Victor G. Coronado (2010) CDC, National Center for Injury Prevention and Control.

3. Teasdale G, Jennett B (1974) Assessment of coma and impaired consciousness. A practical scale. Lancet 2: 81-4.

4. Boone MD, Oren-grinberg A, Robinson TM, Chen CC, Kasper EM (2015) Mannitol or hypertonic saline in the setting of traumatic brain injury: What have we learned? Surg Neurol Int 6: 177.

5. Peters NA, Farrell LB, Smith JP (2018) Hyperosmolar Therapy for the Treatment of Cerebral Edema.US Pharmacist 43: 8-11.

6. Muizelaar JP, Wei EP, Kontos HA, Becker DP (1983) Mannitol causes compensatory cerebral vasoconstriction and vasodilation in response to blood viscosity changes. J Neurosurg 59: 822-8.

7. Carney N, Totten AM, O'reilly C (2017) Guidelines for the Management of Severe Traumatic Brain Injury, Fourth Edition. Neurosurgery 80: 6-15.

\section{Citation:}

Taylor Schwebke, Marc McDowell, Lina Piech, Robert Mokszycki and Ellen Omi (2019) Evaluation of Efficacy of Hypertonic Saline and Mannitol in Combination in Patients with a Traumatic Brain Injury. J Neurol Neurocrit Care Volume 2(1): 1-4. 